\title{
Therapeutic issues with, and long-term outcomes of, pulmonary mycobacterial tuberculosis treatment in patients with autoimmune rheumatic diseases
}

\author{
Dong Won Park, Sung Jun Chung, Yoomi Yeo, Tai Sun Park, Hyun Lee, Ji-Yong Moon, Sang-Heon Kim, \\ Tae-Hyung Kim, Ho Joo Yoon, Jang Won Sohn \\ Department of Internal Medicine, Hanyang University College of Medicine, Seoul, Korea \\ Contributions: (I) Conception and design: DW Park, JW Sohn; (II) Administrative support: None; (III) Provision of study materials or patients: DW \\ Park, SH Kim, HJ Yoon, JW Sohn; (IV) Collection and assembly of data: DW Park, Y Yeo, SJ Chung, TS Park, H Lee, JY Moon; (V) Data analysis \\ and interpretation: DW Park, TH Kim, HJ Yoon; (VI) Manuscript writing: All authors; (VII) Final approval of manuscript: All authors. \\ Correspondence to: Jang Won Sohn, MD, PhD. Department of Internal Medicine, Hanyang University College of Medicine, 222-1 Wangsimni-ro, \\ Seongdong-gu, Seoul 04763, Korea. Email: jwsohn@hanyang.ac.kr.
}

\begin{abstract}
Background: Real-world data on treatment safety and outcome of pulmonary tuberculosis (PTB) in patients with rheumatic diseases (RDs) are scarce. This study explored the therapeutic issues of standard first-line anti-tuberculosis (TB) medication in patients in whom PTB complicated autoimmune RDs.

Methods: Observational, retrospective study was conducted in an intermediate TB burden area, South Korea. We evaluated the safety profile of, and adherence to, standard first-line anti-TB medication in PTB patients with systemic RD and assessed the long-term treatment outcomes, up to 84 months after treatment completion.

Results: We included 37 patients suffering from PTB with RD (case group) and 191 without RD (control group). Rheumatoid arthritis (RA) was the most common RD (24 PTB patients, 64.9\%). The frequency of severe adverse drug reactions (ADRs) was significantly higher in the case group than in the control group (36.1\% vs. $12.5 \%, \mathrm{P}=0.003$ ). Severe gastrointestinal problems were the most commonly observed ADRs, with a high frequency consistently noted in both groups. Changes in first-line anti-TB medication because of severe ADRs were significantly more frequent in the case group, compared with the control group (19.4\% vs. $8.3 \%, \mathrm{P}=0.046)$. No significant between-group difference was evident in terms of long-term unfavorable outcomes (including relapse and mortality) (5.7\% cases vs. $1.2 \%$ controls, $\mathrm{P}=0.146)$.

Conclusions: Clinicians may encounter difficulties when treating PTB in patients with RD. Despite the favorable long-term outcomes of RD patients, the outcomes of individual patients such as those with systemic lupus erythematosus (SLE) should be interpreted with caution during post-therapy follow-up.
\end{abstract}

Keywords: Tuberculosis (TB); pulmonary; rheumatic diseases (RDs); outcome; treatment

Submitted May 16, 2019. Accepted for publication Oct 07, 2019.

doi: $10.21037 /$ jtd.2019.10.74

View this article at: http://dx.doi.org/10.21037/jtd.2019.10.74

\section{Introduction}

Tuberculosis (TB) continues to be a major public health problem worldwide (1). The increased incidence of TB can be attributed to any disorder or immunosuppressive agent that impairs the immune system $(2,3)$. Rheumatic diseases (RDs) such as rheumatoid arthritis (RA), systemic lupus erythematosus (SLE) and ankylosing spondylitis (AS) are characterized by immunological disturbances, and the use of immunosuppressive agents as treatment can further compromise the immune conditions of patients, potentially increasing the risk of TB (4-8). Tumor necrosis factor (TNF) antagonists, important emerging treatment for RD, 
may have contributed to an increase in the occurrence of opportunistic infections, particularly TB $(9,10)$.

The treatment of TB for RD patients follows guidelines identical to those for patients lacking RD. However, those with RD may present with various comorbidities and systemic involvement (11), which may affect both adherence to, and effectiveness of, anti-TB medications $(12,13)$. Moreover, drug-related toxicities and metabolic interactions between immunosuppressive agents and antiTB medications may pose therapeutic challenges (14). Previous studies on TB in patients with RD focused on screening, TB incidence and clinical characteristics, compared to those of the general population (15-19). Little is known on TB treatment safety, adherence to standard regimen, and long-term $\mathrm{TB}$ outcomes in patients with $\mathrm{RD}$. Moreover, common RDs are associated with increased risks of all forms of $\mathrm{TB}$, including both pulmonary tuberculosis (PTB) and extra-pulmonary TB (EPTB) $(16,17)$. Study populations containing considerable proportions of EPTB cases have been researched in terms of how $\mathrm{RD}$ complicates TB $(8,20-22)$.

Thus, in the present observational study, we evaluated the safety profile of, and adherence to, standard first-line anti-TB medication in patients with systemic RD, with a special focus on those with PTB (not EPTB), and assessed the long-term (up to 84 months after treatment completion) outcomes of PTB in patients treated in a single tertiary university-affiliated hospital in South Korea.

\section{Methods}

\section{Study design and subjects}

The medical records of patients with systemic RD and concurrent active PTB treated at Hanyang University Hospital, a tertiary referral hospital in South Korea, between January 12009 and December 31 2011, were retrospectively analyzed according to relevant guidelines and regulations. We included only patients with active PTB, thus excluding those with all other forms of TB including TB pleurisy, hilar and/or lymphadenopathic TB, miliary TB. Those with PTB and concomitant EPTB, or multidrug-resistant TB, were also excluded. During the study period, TB was detected in 56 patients with $\mathrm{RD}$ and 43 of those cases $(76.8 \%)$ had pulmonary involvement (Table S1). Among them, five cases had PTB concomitant with EPTB (pleural TB in three cases, bone/joint involvement of TB in one, and miliary TB in one). A total of 38 patients with active PTB and RD received anti-TB medication. Of these, one with RA had multidrug-resistant $\mathrm{TB}$ and was therefore excluded (Table S2). Ultimately, 37 patients with active PTB and RD comprised the case group.

For comparative purposes, patients with newly diagnosed active PTB, without any evidence of RD treated in our respiratory clinic during the same 3 -year study period served as control group. Patients with HIV infections and active malignancies were excluded from both groups. Patients were followed-up after the end of anti-TB medication for up to 7 years or to the date of censoring (December 31, 2018). When evaluating relapse and other outcomes, we referred to the last date of chest radiography or computed tomography (CT), not the date of the final clinic visit.

\section{Diagnosis of active PTB and RDs}

PTB was diagnosed bacteriologically, pathologically, or clinically. Sputum acid-fast bacilli (AFB) smear/TB cultures were performed at least three times. We subjected bronchoscopic washings to TB cultures only for cases with suspected PTB and negative sputum AFB smear/ TB cultures (23). Active PTB was diagnosed using at least one of the following criteria: (I) AFB organisms evident on sputum AFB smear/TB culture; (II) biopsy-based histological confirmation of a lung lesion, compatible with chronic granulomatous inflammation and caseous necrosis, that stained positive for AFB; and/or (III) typical findings of chest radiographs and/or CT scans combined with a favorable response to anti-TB medication in cases for whom bacteriological or histological confirmation was lacking. Systemic RDs were diagnosed by a board-certified rheumatologist.

\section{Data collection}

For all 228 patients, we collected data on age, sex, comorbid conditions, and any history of TB; radiographic findings; PTB documentation; TB drug sensitivities; and adverse drug reactions (ADRs) during the course of anti-TB medication; treatment outcomes. First of all, two thoracic radiologists who were blinded to all clinical data, interpreted the radiographs and chest CT images, if available. They recorded the presence or absence of typical radiologic TB lesions, which included centrilobular nodules, branching linear and nodular opacities (tree-in-bud sign), patchy or lobular consolidation, and cavitation (24). The 
PTB lesion was graded as minimal, moderately advanced, or far advanced and its location was recorded. Moreover, the images were also assessed for evidence of chronic lung diseases including ILD and others. For patients with RD, we collected the following data: duration of systemic RD, use of immunosuppressive agents including corticosteroids and disease-modifying anti-rheumatic drugs (DMARDs) during the 6 months prior to TB diagnosis, and the use of $\mathrm{TNF}$ antagonists at any time during the course of RD.

\section{Anti-TB medication safety, adberence and long-term treatment outcomes}

Anti-TB medication (self-administered) was initiated after diagnosis of active PTB. The standard first-line treatment consisted of daily isoniazid, rifampicin, pyrazinamide, and/ or ethambutol for the first 2 months (the intensive phase), followed by daily isoniazid, rifampicin, and/or ethambutol for the next 4 months (the continuation phase). The latter phase could be extended for an additional 3 months (to afford a total treatment time of 9 months) for patients with diabetes, those with radiographic cavities, and those who remained smear- or culture-positive at the end of the intensive phase. All treatment durations were at least 6 months. All PTB patients were observed 1 or 2 weeks after commencement of medication, and monthly thereafter, and were asked about any drug side-effects at these visits. However, ADR detection may have differed between the case and control group. During anti-TB treatment, RD patients visited both our respiratory and rheumatology clinics, whereas, those in control group visited only the respiratory clinic. To deal with this limitation, we focused only on serious ADRs, defined as any side-effect that triggered discontinuation of, or change in anti-TB treatment (temporally or permanently), and/or directly caused hospitalization. Drug-induced hepatitis was defined as liver transaminases more than three times higher than the upper limit of normal accompanied by symptoms such as anorexia, nausea, vomiting, or abdominal pain; or more than five times the upper limit in those without symptoms. Mild grade of side-effect that were generally well-tolerated, such as mild elevated levels of transaminase, mild leucopenia $(>3,000$ cells $/ \mu \mathrm{L})$, and gastrointestinal (GI) troubles, were not considered serious ADRs. We monitored treatment completion and adherence to first-line anti-TB treatment by severe ADR status. In terms of long-term outcomes, we recorded relapses, deaths, and acquired drug resistance by whether the treatment regimen had been changed for patients who completed the planned anti-TB medication regimens.

\section{Statistical analysis}

SPSS for Windows (ver. 22.0; SPSS Inc.) was used for all statistical analyses after normal parametric distributions were confirmed using the Kolmogorov-Smirnov test. Continuous variables were compared employing the $t$-test or the nonparametric Mann-Whitney $U$-test, and categorical variables using the chi-squared or Fisher's exact test. All nominal variables are expressed as frequencies. We compared the clinical characteristics and treatment outcomes of PTB patients with and without RD. The RD patients had various types of RD (64.9\% RA) (Table 1). As such skewing could affect the results, we divided the case group into those with RA and those with RDs other than RA; these two subgroups were compared to the control group. All tests were two-sided, and a $\mathrm{P}$ value $<0.05$ was considered to reflect statistical significance.

\section{Ethics statement}

The study protocol was approved by the institutional review board (IRB) of Hanyang University Hospital, Seoul, South Korea (IRB No. 2016-08-036). All data were anonymized before analysis and informed consent from the study participants was waived due to the retrospective nature of this study.

\section{Results}

\section{Baseline characteristics of the study subjects}

Among 37 patients with active PTB and RD, RA was the most frequently reported systemic RD (24 patients, 64.9\%), followed by AS (4 patients, 10.8\%) and SLE (4 patients, $10.8 \%)$. As the control group, we included 191 patients with newly diagnosed active PTB without any evidence of RD. The characteristics of all patients are listed in Table 1 . The mean ages of case and control patients were $50.3 \pm 16.0$ and $47.4 \pm 20.1$ years, respectively $(\mathrm{P}=0.171)$. The case group exhibited a significant female predominance $(\mathrm{P}=0.020)$. Of patients with PTB and RA (the majority of the case group), most were females, and older than those in the control group ( $\mathrm{P}=0.025$ and $\mathrm{P}=0.027$, respectively). We found no significant between-group differences in the frequency of previous TB history, diabetes mellitus, or liver disease 
Table 1 Baseline characteristics of enrolled subjects with active pulmonary tuberculosis

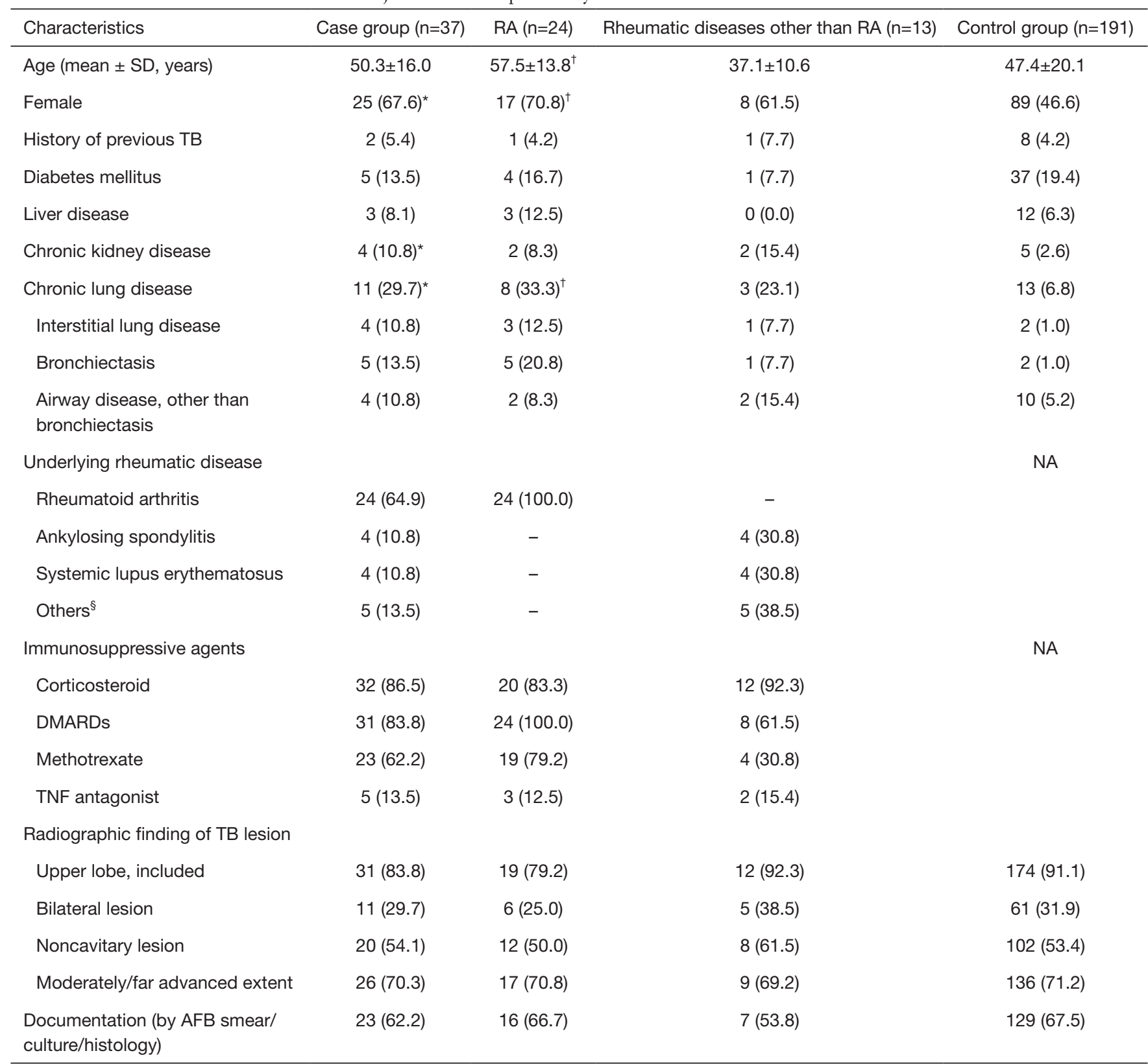

Interstitial lung disease and airway disease (asthma, chronic obstructive pulmonary disease, and bronchiectasis) are included in chronic lung disease and can be detected simultaneously in a single patient. * , ${ }^{\dagger}: \mathrm{P}<0.05$, compared to the control group, using Fisher's exact test, the Mann-Whitney nonparametric $U$-test, the t-test, or the chi-squared test, as appropriate. ${ }^{\S}$ : Adult-onset Still's disease ( $n=1$ ), Behçet's disease $(n=1)$, dermatomyositis $(n=1)$, mixed connective tissue disease $(n=1)$, or systemic sclerosis $(n=1)$. DMARDs, disease-modifying antirheumatic drugs; NA, not applicable; RA, rheumatoid arthritis; SD, standard deviation; TB, tuberculosis; TNF, tumor necrosis factor.

(including hepatitis and cirrhotic liver disease). Patients with RD exhibited a significantly higher frequency of chronic lung diseases, including interstitial lung disease and airway disease (11 patients, $29.7 \%$ ) than did control patients
$(13,6.8 \%, \mathrm{P}<0.001)$; this tendency was consistent for both patients with RA and those with RDs other than RA.

Immunosuppressive agents given prior to $\mathrm{PTB}$ diagnosis included corticosteroids for 32 (86.5\%) 
Table 2 Treatment results and serious adverse drug reactions among pulmonary TB patients who completed anti-tuberculosis medication regimens at our hospital

\begin{tabular}{|c|c|c|c|c|}
\hline Treatment result variables & Case group ( $\mathrm{n}=36)$ & $\mathrm{RA}(\mathrm{n}=23)$ & Rheumatic diseases other than RA $(n=13)$ & Control group $(n=168)$ \\
\hline Radiographic response & $33(91.7)$ & $21(91.3)$ & $12(92.3)$ & $156(92.9)$ \\
\hline \multicolumn{5}{|l|}{ Treatment outcomes } \\
\hline Treatment completion & $35(97.2)$ & $22(95.7)$ & $13(100.0)$ & $162(96.4)$ \\
\hline \multicolumn{5}{|l|}{ Death } \\
\hline TB related & $1(2.8)$ & $1(4.3)$ & $0(0.0)$ & $2(1.2)$ \\
\hline Other related & $0(0.0)$ & $0(0.0)$ & $0(0.0)$ & $4(2.4)$ \\
\hline \multicolumn{5}{|l|}{ Serious adverse drug reaction } \\
\hline Severe GI trouble & $9(25.0)^{*}$ & $5(21.7)^{\dagger}$ & $4(30.8)^{\ddagger}$ & $4(2.4)$ \\
\hline Skin & $2(5.6)$ & $2(8.7)$ & $0(0.0)$ & $2(1.2)$ \\
\hline Hepatotoxicity & $3(8.3)$ & $1(4.3)$ & $2(15.4)$ & $11(6.5)$ \\
\hline Visual changes & $1(2.8)$ & $0(0.0)$ & $1(7.7)$ & $3(1.8)$ \\
\hline Hematologic problem & $1(2.8)$ & $0(0.0)$ & $1(7.7)$ & $1(0.6)$ \\
\hline Renal failure & $1(2.8)$ & $0(0.0)$ & $1(7.7)$ & $0(0.0)$ \\
\hline Others ${ }^{\S}$ & $3(8.3)$ & $1(4.3)$ & $2(15.4)^{\ddagger}$ & $3(1.8)$ \\
\hline Total & $13(36.1)^{\star}$ & $7(30.4)^{\dagger}$ & $6(46.2)^{\ddagger}$ & $21(12.5)$ \\
\hline $\begin{array}{l}\text { Change of } 1 \text { st line anti-TB regimen } \\
\text { due to adverse drug reaction" }\end{array}$ & $7(19.4)^{\star}$ & $2(8.7)$ & $5(38.5)^{\ddagger}$ & $14(8.3)$ \\
\hline
\end{tabular}

${ }^{*},{ }^{\dagger}, \stackrel{\ddagger}{\text { : }} \mathrm{P}<0.05$, compared to the control group, using Fisher's exact test, the Mann-Whitney nonparametric $U$-test, the $t$-test, or the chisquared test, as appropriate. ${ }^{\S}$ : including fever $(n=4)$, shock $(n=1)$, and hyperuricemia $(n=1)$. ^: Patients for whom the anti-tuberculosis regimens were changed among survivors who completed anti-tuberculosis medication courses (22 with RA and 13 with rheumatic diseases other than RA). Gl, gastrointestinal; RA, rheumatoid arthritis; TB, tuberculosis.

and MTX for $23(62.2 \%)$ patients in the case group [corticosteroids for $20(83.3 \%)$ and MTX for 19 (79.2\%) RA patients]. Five patients $(13.5 \%)$ had received TNF antagonists commencing $10-83$ months prior to PTB diagnosis (Table S3). There were no significant betweengroup differences in terms of radiographic features of PTB [upper lobe involvement, bilateral spread, or cavity formation; 218 patients (95.6\% of the 228 ) underwent chest $\mathrm{CT}]$. The diagnostic criteria used to detect PTB were similar in both groups. The drug-resistant TB patterns were also similar in both groups (Table S2).

\section{Treatment outcomes and serious ADRs}

Nine patients of the control group were transferred to another hospital; $1 \mathrm{RA}$ patient in the case group was lost to follow-up, as were 14 in the control group. The treatment results and serious ADRs among the 204 PTB patients who completed anti-TB medication at our hospital (36 in the case group and 168 in the control group) are listed in Table 2. No significant between-group difference in mortality was evident during the course of anti-TB medication. The proportion of patients experiencing serious ADRs was significantly higher in the case $(13,36.1 \%)$ than the control $(21,12.5 \%$ group; $\mathrm{P}=0.003)$. The most common serious ADRs were severe GI problems in the case group and hepatotoxicity in the control group. GI problems were more frequent in the case group (9 patients, $25.0 \%)$ than in the control group (4 patients, $2.4 \%$; $<<0.001$ ); high frequencies of GI problems were noted in both patients with RA and with RDs other than RA. However, no significant between-group difference in the prevalence of hepatotoxicity was evident.

The standard first-line regimen of TB had to be changed to second-line drugs because of drug-induced severe ADRs in 7 (19.4\%) of 36 patients in the case group who 
Table 3 Multivariate logistic regression analysis of clinical factors associated with changed first-line anti-TB drug regimens because of adverse drug reactions in pulmonary TB patients

\begin{tabular}{lc}
\hline Variables & Adjusted OR (95\% Cl) \\
\hline Age, year & 1.000 \\
$<50$ & $0.726(0.177-2.976)$ \\
$50-70$ & $1.725(0.433-6.955)$ \\
$>70$ & \\
Gender & 1.000 \\
Male & $1.221(0.440-3.393)$ \\
Female & 1.000 \\
Underlying rheumatic disease & $0.700(0.122-4.007)$ \\
No rheumatic disease & $6.851(1.694-27.712)$ \\
Rheumatic arthritis & \\
Rheumatic disease other & \\
than rheumatic arthritis & \\
Underlying disease (vs. none) & $0.495(0.093-2.630)$ \\
Diabetes mellitus & $2.856(0.523-15.607)$ \\
Liver disease & $1.070(0.083-13.841)$ \\
Chronic kidney disease & $2.304(0.532-9.974)$ \\
Chronic lung disease &
\end{tabular}

TB, tuberculosis.

completed anti-TB treatment. The frequency of first-line regimen change was significantly higher in the case group than in the control group (14 patients, $8.3 \% ; \mathrm{P}=0.046$ ). In the case group, first-line anti-TB medication was welltolerated in PTB patients with RA, while it was changed more frequently in patients with $\mathrm{RD}$ other than RA (5 patients, $38.5 \%)$ than in the control group $(\mathrm{P}=0.001)$. These five patients included one with dermatomyositis, one with adult-onset Still's disease, one with mixed connective tissue disease, one with Behçet's disease, and one with SLE.

\section{Independent predictors of changes in first-line anti-TB medication}

We performed multivariate analysis seeking predictors of first-line anti-TB medication change caused by severe ADRs in all subjects (Table 3) after adjusting for age, sex, and various clinical factors. RD other than RA was associated with a 6.851 -fold greater risk (95\% CI: 1.694-27.712) of a change in first-line anti-TB medication. RA was not significantly associated with such a change. Age $>70$ years, female gender, and underlying liver, kidney, and chronic lung disease were risk factors for such change, but statistical significance was not attained.

\section{Long-term outcomes in patients who completed anti-TB medication}

The long-term outcomes (relapse, death, and acquired drug resistance) of patients who completed anti-TB medication are shown in Table 4. In the case group, 31 patients (88.6\%) were followed-up for at least 24 months; the mean duration of post-treatment follow-up was $41.6 \pm 21.6$ months. Overall, 33 patients $(94.2 \%$ ) in the case group had favorable outcomes. Despite differences in the duration of followup because of the retrospective study design, we found no significant difference in the rate of unfavorable outcomes (including relapse and death) between the RD (2, 5.7\%) and control $(2,1.2 \%, \mathrm{P}=0.146)$ groups. Of patients for whom the first-line regimen was changed because of severe ADRs, one SLE exhibited PTB relapse sensitive to all first-line anti-TB medications 20 months after treatment completion (four SLE patients included in this study); we recorded no relapses in the control group. Of patients who completed the first-line regimen, one RA patient developed acute respiratory failure unrelated to TB relapse, and two control patients relapsed (with PTB and TB lymphadenopathy). Two AS patients in the case group commenced on a TNF antagonist (etanercept) after completion of antiTB medication. These patients have been treated with etanercept for 6 years (thus to the date of censoring. December 31, 2018) without TB relapse.

\section{Discussion}

We evaluated the safety of, and adherence to, standard firstline anti-TB medication in PTB patients with systemic RD, and the long-term treatment outcomes up to 7 years after treatment completion. We found that treatment of active PTB in patients with RD afforded successful radiological responses and treatment outcomes. However, RD patients with active PTB experienced a higher frequency of severe ADRs caused by anti-TB medications, of which severe GI issues predominated. Around $20 \%$ of PTB patients with RDs of various types had to change from the standard antiTB medication because of severe ADRs; this proportion was significantly greater than that in the control group. RDs other than RA (including SLE) were significantly associated 
Table 4 Long-term outcomes of subjects who had previously completed anti-TB medication regimens by whether the first-line medication regimen was changed

\begin{tabular}{|c|c|c|}
\hline Long-term outcomes & \multicolumn{2}{|c|}{ Completion of expected course } \\
\hline Case group (41.6 \pm 21.6 months) & 7 & 28 \\
\hline Relapses & $1^{*}$ & 0 \\
\hline Death & 0 & $1^{\dagger}$ \\
\hline Control group (25.5 \pm 21.8 months) & 13 & 149 \\
\hline Relapses & 0 & $2^{\ddagger}$ \\
\hline Death & 0 & 0 \\
\hline Acquired drug resistance & 0 & 0 \\
\hline
\end{tabular}

*: one SLE patient experienced pulmonary TB relapse sensitive to all anti-TB drugs. Of patients who completed first-line regimens, ${ }^{\dagger}:$ one patient with RA died and ${ }^{\ddagger}$ : two controls experienced TB relapse (pulmonary TB and TB lymphadenopathy). TB, tuberculosis.

with an inability to tolerate standard anti-TB medication after adjusting for several clinical factors. In contrast, RA patients tolerated anti-TB medications as well as did control subjects. Favorable long-term outcomes were evident in almost all patients with RDs (94.2\%), including those with RA, but one of four SLE patients for whom the first-line regimen was changed because of severe ADRs exhibited PTB relapse 20 months after treatment completion.

Although TB can manifest in any tissue, the lung is both a major port of entry into the body and an important target organ. Prompt PTB diagnosis allows early treatment and limits disease spread in the community. However, early detection of EPTB is often difficult; physicians must proactively consider the possibility of EPTB. In RD patients, early EPTB manifestations can be very similar to the inflammatory reactions of RD (25). PTB is one of the most common forms of TB complicated by $\mathrm{RD}$ (26-28). However, TB in patients with various RDs may vary in terms of EPTB proportions. TB patients with SLE (compared to those with RA) tend to exhibit more frequent extrapulmonary involvement (29). The radiographic manifestations of $\mathrm{PTB}$ patients may reflect this. Yoshinaga et al. (26) reported that $30 \%$ of studied RA patients had EPTB; half had been accidentally diagnosed radiographically, and these exhibited unusual patterns including bilateral and non-cavitary pulmonary lesions. In SLE patients, the prevalence of EPTB is 33-60\%, associated with disseminated PTB lesions exhibiting atypical radiographic patterns $(12,30)$. In this regard, delayed recognition of $\mathrm{TB}$ in $\mathrm{RD}$ patients is common when the prevalence of EPTB is high (22), increasing clinical severity and mortality (31). Moreover, significant differences between PTB and EPTB are apparent in terms of the frequency of adverse events caused by anti-TB drugs (22), this may affect TB treatment outcomes in RD patients (27). The strength of our investigation is that we found that the TB treatment outcomes were good in PTB complicated RD patients, lacking EPTB.

The extent of serious ADRs is important in terms of the effectiveness of anti-TB treatments. It has been suggested that anti-TB drug withdrawal because of an adverse reaction is more probable in RA patients with any form of TB compared to non-RA patients (20). We also found that PTB patients with RD experienced a higher frequency of severe ADRs, the most common of which were severe GI problems in RD patients either with or without RA, perhaps reflecting comorbidities and the concurrent use of multiple drugs. However, the frequency of hepatotoxicity did not differ significantly between patients with and without $\mathrm{RD}$, despite the female predominance in the case group (32). Hernandez-Cruz et al. (12) reported that the hepatotoxicity rate during anti-TB therapy was threefold greater in RD patients than in the general population. However, the cited authors included an EPTB population that might differ from PTB patients in terms of drug tolerance. The prevalence of anti-TB drug-induced hepatotoxicity appears to depend on the definition used (33). We defined hepatotoxicity as a severe ADR that resulted 
in discontinuation of, or a change in, anti-TB drugs, and/ or hospitalization. This may partly explain the similar frequency of drug-induced hepatotoxicity observed in our study and control groups. Further studies using larger numbers of $\mathrm{RD}$ patients are required to clarify these issues.

The treatment outcomes of TB complicated by RD vary by the underlying $\mathrm{RD}$, as well as the proportion of cases with EPTB. Previous studies on TB complicating RA showed no significant differences in either sputum conversion time or in-hospital mortality between RA and non-RA patients $(20,26)$, suggesting that RA is not a significant risk factor for an unfavorable treatment outcome or mortality. In this regard, we found that standard firstline anti-TB treatment was well-tolerated in patients with PTB and RA. All PTB-and-RD patients but one (2.8\%) survived; six patients without RD died (3.6\%). During posttherapeutic follow-up, one patient with and two without RD died. Such favorable treatment outcomes may be explained by the characteristics of the study population, which consisted principally of RA patients.

However, we recorded a significantly higher rate of severe ADRs and a more frequent need to change the firstline regimen in patients with RDs other than RA, compared to the control group. TB patients with SLE exhibited a higher relapse rate than did those with RA (29). A high prevalence of EPTB, and more TB-associated mortality, have been reported in SLE patients with TB (30). In our study, relapse after treatment completion in patients with RD was especially worthy of notice. Of all four PTB patients with SLE, one failed to maintain anti-TB medication because of a severe ADR, and relapsed with PTB after treatment completion. These findings suggest that an underlying RD may affect the ADR incidence and maintenance of first-line anti-TB treatment. However, the impacts of individual RD on treatment outcomes require further elucidation.

Our study had certain limitations. First, PTB lesions can resemble other pulmonary lesions. Lung involvement in RDs may include the lung parenchyma, pleura, vessels and respiratory muscles. The effects of RD on lung parenchyma are largely divided into ILD and airway disease including bronchiolitis, bronchiectasis, and emphysema (34). Bronchiectasis is common in RDs, especially RA, and may increase the risk of infection with RA treatment, especially with TNF antagonists. Bronchiectasis was detected in only 5 cases $(13.5 \%)$, particularly in $20.8 \%$ of RA, four of whom had upper lobe involvement and two had bilateral spread (Table 1). All five cases had confirmed TB and all improved after anti-TB treatment. However, in clinical practice, it may be challenging to differentiate $\mathrm{PTB}$ lesions from lung manifestations of RDs and the nodular, bronchiectatic form of non-TB mycobacterial (NTM). Recent studies have reported an increased risk of NTM infection in RD patients, $(16,21)$, possibly creating clinical confusion, as NTM often mimics TB (35). Moreover, cases of undocumented status, accounting for one third of all included patients, may have been related to NTM infections. Further research with more documented cases is required. Second, we only included cases of PTB, not EPTB. We found that 26 of 30 TB patients with RA $(86.7 \%)$ had PTB (among them, one case had both PTB and EPTB in the bones/joints). All six TB patients with AS had PTB, and two of them had both PTB and TB pleurisy. On the other hand, 12 TB cases were identified in SLE, of which only $5(41.7 \%)$ were found to have PTB (Table S1). The incidence of $\mathrm{PTB}$ in patients with RDs varies depending on the RD. Since this study included a significant portion of RA patients, a large portion of all TB patients with RDs may have PTB. However, our study focused exclusively on PTB, not on all cases of TB including EPTB. Therefore, it may not be possible to generalize our results to all TB patients including those with EPTB. Third, most RD patients were regularly followed-up in outpatient clinics, and latent TB infection was occasionally checked via chest radiography prior to commencement of TNF antagonists. Such differences between the two groups might have affected disease severity and treatment outcomes. Fourth, our study was performed in a single-institution based study design and included a relatively small sample size, which may have led to potential bias in patient selection. Finally, our study was also limited by its retrospective nature; we had information only on severe ADRs. Thus, our data on ADR frequency should be interpreted with caution.

\section{Conclusions}

In conclusion, we found that patients in whom active PTB complicated RD experienced a high rate of severe ADRs, predominantly GI issues. Around 20\% of those with all types of $\mathrm{RD}$ required changes to the standard first-line anti-TB medication because of severe ADRs; however, RA patients tolerated anti-TB medications as well as did control subjects. Although the long-term outcomes were favorable in almost all RD patients, including those with RA, the 
outcomes of those with certain RDs (such as SLE) during post-therapy follow-up should be interpreted with caution.

\section{Acknowledgments}

Funding: This study was supported by the research fund of Hanyang University (HY-201400000003104).

\section{Footnote}

Conflicts of Interest: The authors have no conflicts of interest to declare.

Ethical Statement: The authors are accountable for all aspects of the work in ensuring that questions related to the accuracy or integrity of any part of the work are appropriately investigated and resolved. The study protocol was approved by the Institutional Review Board of Hanyang University Hospital, Seoul, South Korea (IRB No. 201608-036). All data were anonymized before analysis and informed consent from the study participants was waived due to the retrospective nature of this study.

\section{References}

1. American Thoracic Society; Centers for Disease Control and Prevention; Infectious Diseases Society of America. American Thoracic Society/Centers for Disease Control and Prevention/Infectious Diseases Society of America: controlling tuberculosis in the United States. Am J Respir Crit Care Med 2005;172:1169-227.

2. Bass JB Jr, Farer LS, Hopewell PC, et al. Treatment of tuberculosis and tuberculosis infection in adults and children. American Thoracic Society and The Centers for Disease Control and Prevention. Am J Respir Crit Care Med 1994;149:1359-74.

3. Kobashi Y, Matsushima T. Clinical analysis of pulmonary tuberculosis in association with corticosteroid therapy. Intern Med 2002;41:1103-10.

4. Kim EM, Uhm WS, Bae SC, et al. Incidence of tuberculosis among korean patients with ankylosing spondylitis who are taking tumor necrosis factor blockers. J Rheumatol 2011;38:2218-23.

5. Marie I, Hachulla E, Cherin P, et al. Opportunistic infections in polymyositis and dermatomyositis. Arthritis Rheum 2005;53:155-65.

6. Tam LS, Li EK, Wong SM, et al. Risk factors and clinical features for tuberculosis among patients with systemic lupus erythematosus in Hong Kong. Scand J Rheumatol 2002;31:296-300.

7. van Ingen J, Boeree MJ, Dekhuijzen PN, et al. Mycobacterial disease in patients with rheumatic disease. Nat Clin Pract Rheumatol 2008;4:649-56.

8. Chang YS, Liu CJ, Ou SM, et al. Tuberculosis infection in primary Sjogren's syndrome: a nationwide populationbased study. Clin Rheumatol 2014;33:377-83.

9. Psichogiou M, Sfikakis PP, Kontos F, et al. Tuberculosis after anti-TNF therapy: a continuous learning process. Scand J Rheumatol 2012;41:401-3.

10. Seong SS, Choi CB, Woo JH, et al. Incidence of tuberculosis in Korean patients with rheumatoid arthritis (RA): effects of RA itself and of tumor necrosis factor blockers. J Rheumatol 2007;34:706-11.

11. Emamifar A, Levin K, Jensen Hansen IM. Patients with newly diagnosed rheumatoid arthritis are at increased risk of diabetes mellitus: an observational cohort study. Acta Reumatol Port 2017;42:310-7.

12. Hernández-Cruz B, Sifuentes-Osornio J, Ponce-de-León Rosales S, et al. Mycobacterium tuberculosis infection in patients with systemic rheumatic diseases. A case-series. Clin Exp Rheumatol 1999;17:289-96.

13. Kaufmann SH. How can immunology contribute to the control of tuberculosis? Nat Rev Immunol 2001;1:20-30.

14. Yew WW. Clinically significant interactions with drugs used in the treatment of tuberculosis. Drug Saf 2002;25:111-33.

15. Huang LK, Wu MH, Chang SC. Radiological manifestations of pulmonary tuberculosis in patients subjected to anti-TNF-alpha treatment. Int J Tuberc Lung Dis 2014;18:95-101.

16. Yeh JJ, Wang YC, Sung FC, et al. Rheumatoid arthritis increases the risk of nontuberculosis mycobacterial disease and active pulmonary tuberculosis. PLoS One 2014;9:e110922.

17. Lu MC, Lai CL, Tsai CC, et al. Increased risk of pulmonary and extra-pulmonary tuberculosis in patients with rheumatic diseases. Int J Tuberc Lung Dis 2015;19:1500-6.

18. Shobha V, Chandrashekara S, Rao V, et al. Biologics and risk of tuberculosis in autoimmune rheumatic diseases: A real-world clinical experience from India. Int J Rheum Dis 2019;22:280-7.

19. Wang X, Wong SH, Wang XS, et al. Risk of tuberculosis in patients with immune-mediated diseases on biological therapies: a population-based study in a tuberculosis endemic region. Rheumatology (Oxford) 2019;58:803-10. 
20. Ishiguro T, Takayanagi N, Kagiyama N, et al. Characteristics of Tuberculosis in Patients with Rheumatoid Arthritis: A Retrospective Single-center Study. Internal Medicine 2014;53:1291-8.

21. Brode $\mathrm{SK}$, Jamieson $\mathrm{FB}, \mathrm{Ng} \mathrm{R}$, et al. Increased risk of mycobacterial infections associated with anti-rheumatic medications. Thorax 2015;70:677-82.

22. Hou CL, Tsai YC, Chen LC, et al. Tuberculosis infection in patients with systemic lupus erythematosus: pulmonary and extra-pulmonary infection compared. Clin Rheumatol 2008;27:557-63.

23. Iyer VN, Joshi AY, Boyce TG, et al. Bronchoscopy in suspected pulmonary TB with negative induced-sputum smear and MTD((R)) Gen-probe testing. Respir Med 2011;105:1084-90.

24. Jeong YJ, Lee KS. Pulmonary tuberculosis: up-todate imaging and management. AJR Am J Roentgenol 2008;191:834-44.

25. Lawn SD, Zumla AI. Tuberculosis. Lancet 2011;378:57-72.

26. Yoshinaga Y, Kanamori T, Ota Y, et al. Clinical characteristics of Mycobacterium tuberculosis infection among rheumatoid arthritis patients. Mod Rheumatol 2004;14:143-8.

27. Erdozain JG, Ruiz-Irastorza G, Egurbide MV, et al. High risk of tuberculosis in systemic lupus erythematosus? Lupus 2006;15:232-5.

28. Baronnet L, Barnetche T, Kahn V, et al. Incidence of tuberculosis in patients with rheumatoid arthritis. A systematic literature review. Joint Bone Spine 2011;78:279-84.

29. Yun JE, Lee SW, Kim TH, et al. The incidence and clinical characteristics of Mycobacterium tuberculosis infection among systemic lupus erythematosus and rheumatoid arthritis patients in Korea. Clin Exp Rheumatol 2002;20:127-32.

30. Sayarlioglu M, Inanc M, Kamali S, et al. Tuberculosis in Turkish patients with systemic lupus erythematosus: increased frequency of extrapulmonary localization. Lupus 2004;13:274-8.

31. Virenfeldt J, Rudolf F, Camara C, et al. Treatment delay affects clinical severity of tuberculosis: a longitudinal cohort study. BMJ Open 2014;4:e004818.

32. Yee D, Valiquette C, Pelletier M, et al. Incidence of serious side effects from first-line antituberculosis drugs among patients treated for active tuberculosis. Am J Respir Crit Care Med 2003;167:1472-7.

33. Saukkonen JJ, Cohn DL, Jasmer RM, et al. An official ATS statement: hepatotoxicity of antituberculosis therapy. Am J Respir Crit Care Med 2006;174:935-52.

34. Doyle TJ, Dellaripa PF. Lung Manifestations in the Rheumatic Diseases. Chest 2017;152:1283-95.

35. Johnson MM, Odell JA. Nontuberculous mycobacterial pulmonary infections. J Thorac Dis 2014;6:210-20.

Cite this article as: Park DW, Chung SJ, Yeo Y, Park TS, Lee H, Moon JY, Kim SH, Kim TH, Yoon HJ, Sohn JW. Therapeutic issues with, and long-term outcomes of, pulmonary mycobacterial tuberculosis treatment in patients with autoimmune rheumatic diseases. J Thorac Dis 2019;11(11):45734582. doi: $10.21037 / j t d .2019 .10 .74$ 


\section{Supplementary}

Table S1 Frequency of pulmonary and extrapulmonary tuberculosis in patients with rheumatic diseases

\begin{tabular}{|c|c|c|c|c|c|c|c|c|c|}
\hline Site of tuberculosis & RA & SLE & AS & $\mathrm{DM}$ & SSc & Behcet & MCTD & AOSD & Total \\
\hline Pleuritis and empyema & 2 & 2 & 2 [2] & $1[1]$ & & & & & $7[3]$ \\
\hline Lymph nodes & 1 & 2 & & & & & & & 3 \\
\hline Bone and joint & & $1[1]$ & & 1 & & & & & $2[1]$ \\
\hline Miliary & $1[1]$ & & & 1 & & & & & $2[1]$ \\
\hline Meningitis & 1 & 1 & & & & & & & 2 \\
\hline Total & $30[1]$ & $12[1]$ & 6 [2] & $4[1]$ & 1 & 1 & 1 & 1 & $56[5]$ \\
\hline
\end{tabular}

The patients with pulmonary tuberculosis (TB) and concomitant extrapulmonary TB in those with rheumatic diseases are presented in square brackets. *, one patient with RA who had pulmonary multi-drug resistant TB. AOSD, adult-onset still's disease; AS, ankylosing spondylitis; DM, dermatomyositis; MCTD, mixed connective tissue disease; RA, rheumatoid arthritis; SLE, systemic lupus erythematosus; SSc, systemic sclerosis; TB, tuberculosis.

Table S2 Drug resistance patterns of all patients with positive TB cultures.

\begin{tabular}{lcccc}
\hline Drug resistance patterns & Case group & RA & Rheumatic diseases other than RA & Control group \\
\hline Sensitive to INH and RFP & 10 & 6 & 4 & 1 \\
Sensitive to RFP, but resistant to INH & 1 & 0 & 0 & 60 \\
Sensitive to INH, but resistant to RFP & 0 & 1 & 0 & 1 \\
Resistant to INH and RFP* & 1 & 4 & 6 \\
Not checked & 5 & 11 & 21 \\
Total & 17 & 120 \\
\hline
\end{tabular}

*, excluding three patients resistant to INH and RFP. INH, isoniazid; RFP, rifampin; TB, tuberculosis.

Table S3 Clinical characteristics of patients with active pulmonary TB who also had rheumatic diseases treated using anti-TNF antagonists

\begin{tabular}{|c|c|c|c|c|c|}
\hline Characteristic & Patient 1 & Patient 2 & Patient 3 & Patient 4 & Patient 5 \\
\hline $\begin{array}{l}\text { Underlying rheumatic } \\
\text { disease }\end{array}$ & Rheumatoid arthritis & Rheumatoid arthritis & Rheumatoid arthritis & Ankylosing spondylitis & $\begin{array}{c}\text { Adult onset Still's } \\
\text { disease }\end{array}$ \\
\hline Disease duration (years) & 18 & 8 & 3 & 8 & 2 \\
\hline $\begin{array}{l}\text { Old pulmonary TB lesion } \\
\text { on chest radiograph }\end{array}$ & - & - & - & + & - \\
\hline $\begin{array}{l}\text { Underlying pulmonary } \\
\text { disease }\end{array}$ & Bronchiectasis & - & - & - & - \\
\hline
\end{tabular}

Table S3 (continued) 
Table S3 (continued)

\begin{tabular}{|c|c|c|c|c|c|}
\hline Characteristic & Patient 1 & Patient 2 & Patient 3 & Patient 4 & Patient 5 \\
\hline Baseline TST & - & + & - & + & - \\
\hline Baseline IGRA & ND & ND & ND & + & ND \\
\hline Anti-TNF agents used & Adalimumab & Golimumab & Etanercept & Adalimumab & $\begin{array}{c}\text { Adalimumab, } \\
\text { infliximab, } \\
\text { etanercept }\end{array}$ \\
\hline LTBI treatment & ND & INH 9m & ND & INH 9m & ND \\
\hline $\begin{array}{l}\text { Time to pulmonary TB } \\
\text { after start of anti-TNF } \\
\text { agents, months }\end{array}$ & 83 & 36 & 17 & 13 & 10 \\
\hline Relapse & No & No & No & No & No \\
\hline
\end{tabular}

*, etanercept was recommenced 5 months after completion of TB medication. CYC, cyclophosphamide; DMARDs, disease-modifying antirheumatic drugs; IGRA, interferon-gamma release assay; IVIG, intravenous immunoglobulin; LEF, Leflunomide; MTX, methotrexate; ND, not done; TB, tuberculosis; TNF, tumor necrosis factor; TST, tuberculin skin test. 